OCTOBER, 1974

\title{
STABILITY OF PURE HOMOGENEOUS DEFORMATIONS OF AN ELASTIC CUBE UNDER DEAD LOADING*
}

\author{
BY
}

\author{
R. S. RIVLIN
}

Lehigh University

1. Introduction. In a previous paper [1], the problem was considered of the pure homogeneous deformation of a unit cube of incompressible neo-Hookean elastic material by three pairs of equal and opposite forces acting normally on the faces of the cube and distributed uniformly over them. It was found that, for certain specified values of the forces, more than one equilibrium state of pure homogeneous deformation can exist. The stability of each of these states was investigated, with respect to superposed infinitesimal pure homogeneous deformations, with the same principal directions as the equilibrium state. It was found that for certain ranges of values of the applied forces, more than one equilibrium state of pure homogeneous deformation which is stable in this sense can exist. Which of these stable states is actually attained in practice will depend on the order in which the forces are applied.

As a special case, the situation was considered in which all three pairs of forces are the same and it was found that, even in this case, for certain values of the applied forces, more than one stable equilibrium state is possible. We denote by $\lambda_{1}, \lambda_{2}, \lambda_{3}$ the extension ratios for the pure homogeneous deformation and by $W$ the strain-energy per unit volume. Then, for an incompressible neo-Hookean material, $W$ is given by

$$
W=\frac{1}{2} C\left({\lambda_{1}}^{2}+{\lambda_{2}}^{2}+{\lambda_{3}}^{2}-3\right) \text {, }
$$

where $C$ is a positive constant. Let $T$ be the force applied to each face of the cube and we take $T$ to be positive if the force is directed outwards from the cube. It was found by Rivlin [1] that if

$$
T / C<3\left(\frac{1}{4}\right)^{1 / 3}
$$

only one equilibrium state is possible, that for which $\lambda_{1}=\lambda_{2}=\lambda_{3}=1$ and this state is stable in the sense described above. If

$$
3\left(\frac{\mathbf{1}}{4}\right)^{1 / 3}<T / C<2,
$$

then seven equilibrium states are possible, four of them being stable in the sense described above and three of them being unstable. One of the stable equilibrium states is that for which $\lambda_{1}=\lambda_{2}=\lambda_{3}=1$ and the other three have two principal extension ratios equal and different from unity. These three states are the same, apart from cyclic permutation of the roles of $\lambda_{1}, \lambda_{2}$ and $\lambda_{3}$. Also, in each of the three unstable equilibrium states, two

* Received March 28, 1973. This work was carried out with the support of the Office of Naval Research under Contract No. N00014-67-0370-0001 with Lehigh University. 
of the extension ratios are equal and different from unity. Again, they are the same apart from cyclic permutation of the roles of $\lambda_{1}, \lambda_{2}$ and $\lambda_{3}$. For $T / C>2$, it was found that there are again seven equilibrium states. However, in this case, three of the states are stable, in the sense described above, and four are unstable. Each of the stable states has two extension ratios equal and different from unity, the states being the same, apart from cyclic permutation of the roles of $\lambda_{1}, \lambda_{2}$ and $\lambda_{3}$. One of the unstable states is the state for which $\lambda_{1}=\lambda_{2}=\lambda_{3}=1$ and the other three are again states for which two principal extension ratios are equal and different from unity. These three states are the same, apart from cyclic permutation of the roles of $\lambda_{1}, \lambda_{2}$ and $\lambda_{3}$.

Hill [2] discussed the state $\lambda_{1}=\lambda_{2}=\lambda_{3}=1$ in the case of a neo-Hookean material and found that the state is unstable when $T / C>2$ and stable when $0<T / C<2$, in agreement with Rivlin [1].* Hill's stability critcrion is, however, stronger than that of Rivlin, since he required stability with respect to arbitrary infinitesimal superposed deformations. Hill further indicated that the analysis can also be applied in the case of a Mooney material, with an appropriate change in the upper stability limit on $T$. Green and Adkins [3] obtained similar results for the case of a general incompressible isotropic elastic material. Beatty [4] drew particular attention to the instability of the undeformed state when $T<0$, and pointed out that this arises essentially from instability with respect to rigid-body rotations, and accordingly could never have been discovered by using the weaker stability criterion of Rivlin. This result can be read off quite easily from the results of Hill and Green and Adkins.

In the present paper, we discuss the stability of all the equilibrium states which can result from the application, normally to the faces of the cube, of three similar pairs of equal and opposite, uniformly distributed forces $T$. The cube is assumed to consist of an incompressible neo-Hookean material. The stability criterion used here is that used in $[2,3,4]$. It has already been pointed out that this is stronger than the criterion used in [1]. It is, however, found that the stability limits remain the same as those found in [1], with the exception of the instability already mentioned when $\lambda_{1}=\lambda_{2}=\lambda_{3}$ and $T<0$.

2. The stability criterion. We consider the deformation of a body of incompressible elastic material in which a particle initially at $X_{A}$, in the rectangular cartesian coordinate system $x$, moves to $x_{i}$. The deformation is described by the dependence of $x_{i}$ on $X_{A}$ thus:

$$
x_{i}=x_{i}\left(X_{A}\right) .
$$

Since the material is incompressible, the deformation gradients $x_{i, A}$ must satisfy the relation

$$
\left|x_{i, A}\right|=1
$$

We now suppose that the deformation (2.1) results from the superposition of an arbitrary deformation on a homogeneous deformation in which a particle initially at $X_{A}$ moves to $c_{i A} X_{A}$. We accordingly rewrite (2.1) as

$$
x_{i}=c_{i A} X_{A}+u_{i}\left(X_{A}\right)
$$

* Hill [2] noted an apparent conflict of his result, that the undeformed state is unstable when $T / C>2$, with that of Rivlin [1]. This apparent conflict probably results from an error in the abstract of [1], which does not correctly reflect the conclusions in the body of the text, as was pointed out by Beatty [4]. 
where $c_{i A}$ are constants and $u_{i}$ is the displacement associated with the arbitrary superposed deformation.

Let $\Pi_{A i}$ be the Piola-Kirchoff stress associated with the state of homogeneous deformation. We define a functional $\Phi$ of the deformation gradient field $x_{i, A}\left(X_{B}\right)$ by

$$
\Phi=\int_{V}\left[W\left(x_{i, A}\right)-\Pi_{A i} x_{i, A}\right] d V,
$$

where $W$ is the strain-energy per unit volume and $V$ is the domain occupied by the body in the undeformed state. The homogeneous equilibrium states corresponding to a specified dead-loading condition, i.e. to specified values of $\Pi_{A i}$, are those for which $\Phi$ has stationary values with respect to all possible infinitesimal values of the deformation gradients $u_{i, A}$ compatible with the constraint (2.1). The equilibrium states are accordingly given by

$$
\delta \Phi=0
$$

for all allowable infinitesimal $u_{i, A}$.

An equilibrium state is stable if the corresponding stationary value of $\Phi$ is a minimum, i.e. if

$$
\delta^{2} \Phi>0
$$

for all allowable infinitesimal $u_{i, A}$. If this condition is violated the equilibrium state is unstable. (We neglect the case of neutral stability in which $\delta^{2} \Phi$ may be zero for some allowable values of $u_{i, A}$, but is never negative.) The conditions (2.6) and (2.7) for stable equilibrium express the requirement that the work done by the specified forces, in any allowable infinitesimal deformation from a stable equilibrium state, is greater than the increase in strain-energy associated with this deformation.

Following Beatty [4], we can relax the constraint (2.2) on $x_{i, A}$ by using the method of undetermined multipliers. Accordingly, we replace the expression (2.4) for $\Phi$ by

$$
\Phi=\int_{V}\left[W\left(x_{i, A}\right)-\Pi_{A i} x_{i, A}-p\left(\left|x_{i, A}\right|-1\right)\right] d V,
$$

where $p$ is an undetermined multiplier which may depend on $X_{A}$, but is independent of $x_{i, A}$. We note that, for infinitesimal $u_{i, A}$,

$$
\delta\left(\left|x_{i, A}\right|-1\right)=\frac{1}{2} \epsilon_{i j k} \epsilon_{A B C} c_{j B} c_{k C} u_{i, A}=0 .
$$

Thus, (2.7) yields, in agreement with Beatty [4],

$$
\delta \Phi=\int_{V}\left[\frac{\partial W}{\partial c_{i A}}-\Pi_{A i}-\frac{1}{2} p \epsilon_{i j k} \epsilon_{A B C} c_{i B} c_{k C}\right] u_{i, A} d V
$$

and

$$
\delta^{2} \Phi=\frac{1}{2} \int_{V}\left[\frac{\partial^{2} W}{\partial c_{i A} \partial c_{i B}}-p \epsilon_{i j k} \epsilon_{A B} c_{k C}\right] u_{i, A} u_{i, B} d V
$$

where

$$
\frac{\partial W}{\partial c_{i A}}=\left.\frac{\partial W}{\partial x_{i, A}}\right|_{u_{i}=0}, \quad \frac{\partial^{2} W}{\partial c_{i A} \partial c_{i B}}=\left.\frac{\partial^{2} W}{\partial x_{i, A} \partial x_{i, B}}\right|_{u_{i}=0} .
$$


From (2.5) and (2.9) we obtain the well-known formula for the Piola-Kirchoff stress in an incompressible material,

$$
\Pi_{A i}=\left(\partial W / \partial c_{i A}\right)-\frac{i}{2} p \epsilon_{i j k} \epsilon_{A B C} c_{i B} c_{k C} .
$$

For an incompressible neo-Hookean material, the strain-energy $W$ is given by

$$
W=\frac{1}{2} C\left(x_{i, A} x_{i, B}-3\right),
$$

where $C$ is a positive constant. Eq. (2.12) then becomes

$$
\Pi_{A i}=C c_{i A}-\frac{1}{2} p \epsilon_{i j k} \epsilon_{A B C} c_{i B} c_{k C},
$$

and from (2.10) we obtain

$$
\delta^{2} \Phi=\frac{1}{2} \int_{V}\left(C \delta_{i i} \delta_{A B}-p \epsilon_{i j k} \epsilon_{A B C} c_{k C}\right) u_{i, A} u_{i, B} d V .
$$

3. Pure homogeneous deformation. We now suppose that a state of pure homogeneous deformation is produced in a unit cube by three pairs of equal and opposite forces $T$ applied to the faces of the cube. The Piola-Kirchoff stress in the cube is then given by

$$
\Pi_{i A}=T \delta_{i A} .
$$

Suppose the principal extension ratios for the pure homogeneous deformation are $\lambda_{1}, \lambda_{2}, \lambda_{3}$ and the principal directions are parallel to the axes of the reference system $x$. The incompressibility condition (2.2) then yields

$$
\lambda_{1} \lambda_{2} \lambda_{3}=1 \text {. }
$$

From (2.14) and (3.1) we obtain, with (3.2),

$$
T=C \lambda_{1}-p / \lambda_{1}, \quad T=C \lambda_{2}-p / \lambda_{2}, \quad T=C \lambda_{3}-p / \lambda_{3} .
$$

These equations yicld the following results:

$$
\begin{array}{llll}
\text { either } \lambda_{2}=\lambda_{3} & \text { or } & T / C=\lambda_{2}+\lambda_{3}, \\
\text { either } \lambda_{3}=\lambda_{1} & \text { or } & T / C=\lambda_{3}+\lambda_{1}, \\
\text { either } \lambda_{1}=\lambda_{2} & \text { or } & T / C=\lambda_{1}+\lambda_{2} .
\end{array}
$$

Eqs. (3.4), (3.5), (3.6) and (3.2) yield the following possible solutions corresponding to a specified value of $T$ :

$$
\begin{aligned}
\lambda_{1}=\lambda_{2}=\lambda_{3} & =1, \\
\lambda_{2}=\lambda_{3} & \text { and } T / C=\lambda_{1}+\lambda_{2}, \\
\lambda_{3}=\lambda_{1} & \text { and } \quad T / C=\lambda_{2}+\lambda_{3}, \\
\lambda_{1}=\lambda_{2} & \text { and } \quad T / C=\lambda_{3}+\lambda_{1} .
\end{aligned}
$$

(We note that if the second of the two alternatives are taken in each of the equations (3.4), (3.5) and (3.6), we obtain the solution (3.7).) 
In the case (3.10), we obtain from (3.2) the equation

$$
F\left(\lambda_{3}\right)=0 \text {, }
$$

where

$$
F\left(\lambda_{3}\right)=\lambda_{3}\left(\frac{T}{C}-\lambda_{3}\right)^{2}-1 .
$$

The cases (3.8) and (3.9) yield similar equations with $\lambda_{3}$ replaced by $\lambda_{1}$ and $\lambda_{2}$ respectively. We now determine the values of $T / C$ for which the cubic equation (3.11) has one or three real roots for $\lambda_{3}$.

The stationary values of $F\left(\lambda_{3}\right)$ occur when $\lambda_{3}=\frac{1}{3} T / C$ and $\lambda_{3}=T / C$. We note that

$$
\begin{gathered}
F(0)=-1, \quad F\left(\frac{1}{3} \frac{T}{C}\right)=\frac{4}{27}\left(T^{3} / C^{3}\right)-1, \\
F(T / C)=-1, \quad F(\infty)=\infty, \quad F(-\infty)=-\infty .
\end{gathered}
$$

Accordingly, if $T / C>0$, Eq. (3.11) has three positive solutions provided that $T / C>$ $3\left(\frac{1}{4}\right)^{1 / 3}$ and one positive solution if $T / C<3\left(\frac{1}{4}\right)^{1 / 3}$. If the three positive solutions are denoted by $\lambda_{3}{ }^{(1)}, \lambda_{3}{ }^{(2)}, \lambda_{3}{ }^{(3)}$, they must satisfy the inequalities

$$
0<\lambda_{3}{ }^{(1)}<\frac{1}{3} T / C<\lambda_{3}{ }^{(2)}<T / C<\lambda_{3}{ }^{(3)}<\infty .
$$

If only one positive solution exists, it must satisfy the inequality

$$
\lambda_{3}>T / C \text {. }
$$

We discard this solution and the solution $\lambda_{3}{ }^{(3)}$ in (3.14) since, from $(3.10)_{2}$, they lead to $\lambda_{1}<0$.

Turning now to the case when $T / C<0$, we note that there is only one positive solution of (3.11) for $\lambda_{3}$. For this solution $T / C-\lambda_{3}<0$ and hence, from $(3.10)_{2}, \lambda_{1}<0$. We accordingly discard it.

Summarizing the conclusions of this section, we see that

(a) if $T / C<0$, only one equilibrium state is possible, that for which $\lambda_{1}=\lambda_{2}=$ $\lambda_{3}=1$;

(b) if $T / C>0$, seven equilibrium states are possible and these are characterized by extension ratios satisfying the following conditions:

(i) $\lambda_{1}=\lambda_{2}=\lambda_{3}=1$,

(ii) $\lambda_{1}=\lambda_{2}, \quad 0<\lambda_{3}<\frac{1}{3} T / C$,

(iii) $\lambda_{2}=\lambda_{3}, \quad 0<\lambda_{1}<\frac{1}{3} T / C$,

(iv) $\lambda_{3}=\lambda_{1}, \quad 0<\lambda_{2}<\frac{1}{3} T / C$,

(v) $\lambda_{1}=\lambda_{2}, \quad T / C>\lambda_{3}>\frac{1}{3} T / C$,

(vi) $\lambda_{2}=\lambda_{3}, \quad T / C>\lambda_{1}>\frac{1}{3} T / C$,

(vii) $\lambda_{3}=\lambda_{1}, \quad T / C>\lambda_{2}>\frac{1}{3} T / C$.

In the next section, we investigate the stability of these states with respect to superposed infinitesimal deformations.

In the previous paper [1], the results obtained in this section were derived as a special case of the more general problem, in which the three pairs of forces are not necessarily the same. In the present paper, we attain a considerable simplification of the argument leading to these results by introducing $a b$ initio the condition that the three pairs of forces are the same. 
4. Stability of the states of pure homogeneous deformation. We have seen in Sec. 2 that a homogeneous state of deformation is stable, in an incompressible neo-Hookean material, with respect to arbitrary superposed infinitesimal deformations, if

$$
\delta^{2} \Phi>0
$$

where $\Phi$ is given by (2.15). Using (3.3) to substitute for $p$ in (2.15), and introducing

$$
c_{11}=\lambda_{1}, \quad c_{22}=\lambda_{2}, \quad c_{33}=\lambda_{3}, \quad c_{k C}=0 \quad(k \neq C),
$$

we obtain

$$
\begin{aligned}
& \delta^{2} \Phi=\frac{1}{2} C \int_{V}\left\{\left(u_{1,1}\right)^{2}+\left(u_{2,2}\right)^{2}+\left(u_{3,3}\right)^{2}\right. \\
&-2{\lambda_{1}}^{2}\left(\lambda_{1}-T / C\right) u_{2,2} u_{3,3} \\
&-2{\lambda_{2}}^{2}\left(\lambda_{2}-T / C\right) u_{3,3} u_{1,1} \\
&-2{\lambda_{3}}^{2}\left(\lambda_{3}-T / C\right) u_{1,1} u_{2,2} \\
&+ {\left[\left(u_{2,3}\right)^{2}+\left(u_{3,2}\right)^{2}+2{\lambda_{1}}^{2}\left(\lambda_{1}-T / C\right) u_{2,3} u_{3,2}\right] } \\
&+ {\left[\left(u_{3,1}\right)^{2}+\left(u_{1,3}\right)^{2}+2{\lambda_{2}}^{2}\left(\lambda_{2}-T / C\right) u_{3,1} u_{1,3}\right] } \\
&+ {\left.\left[\left(u_{1,2}\right)^{2}+\left(u_{2,1}\right)^{2}+2{\lambda_{3}}^{2}\left(\lambda_{3}-T / C\right) u_{1,2} u_{2,1}\right]\right\} d V . }
\end{aligned}
$$

In the case when $\lambda_{1}=\lambda_{2}=\lambda_{3}=1$, Eq. (4.3) can be rewritten as

$$
\begin{aligned}
\delta^{2} \Phi=\frac{1}{2} C \int_{V}\left\{-\left(1-\frac{T}{C}\right)\left(u_{A, A}\right)^{2}\right. & +\left(2-\frac{T}{C}\right) u_{B, A} u_{B, A} \\
& \left.-\left(1-\frac{T}{C}\right)\left(u_{B, A}-u_{A, B}\right)\left(u_{B, A}-u_{A, B}\right)\right\} d V .
\end{aligned}
$$

The constraint (2.8) on $u_{i, A}$ due to the incompressibility of the material now takes the form

$$
u_{A, A}=0 .
$$

It follows from (4.4) and (4.1), that the condition for stability is

$$
\int_{V}\left\{\left(2-\frac{T}{C}\right) u_{B, A} u_{B, A}-\left(1-\frac{T}{C}\right)\left(u_{B, A}-u_{B, A}\right)\left(u_{B, A}-u_{A, B}\right)\right\} d V>0,
$$

for all $u_{B, A}$ satisfying (4.5). This condition is not satisfied if $T / C>2$ and accordingly, for such values of $T$, the state $\lambda_{1}=\lambda_{2}=\lambda_{3}=1$ is unstable. If $0<T / C<2$, the condition is satisfied and accordingly the state is stable with respect to arbitrary superposed infinitesimal deformations. If $T / C<0$, the condition (4.6) is not satisfied and accordingly, for negative values of $T$, the state $\lambda_{1}=\lambda_{2}=\lambda_{3}=1$ is unstable, as is evident from [2,3,4].

We now consider the cases when (3.10) applies, so that $\lambda_{3}$ is given by (3.11). By using (3.10) and (3.2), we obtain, in this case,

$$
\lambda_{1}{ }^{2}\left(\frac{T}{C}-\lambda_{1}\right)=\lambda_{2}^{2}\left(\frac{T}{C}-\lambda_{2}\right)=1 .
$$

Introducing (4.7) into (4.3), we obtain, with (3.10) and (3.2), 


$$
\begin{gathered}
\delta^{2} \Phi=\frac{1}{2} C \int_{V}\left\{\left(u_{1,1}+u_{2,2}+u_{3,3}\right)^{2}-2\left(1-\lambda_{1} \lambda_{3}{ }^{2}\right) u_{1,1} u_{2,2}\right. \\
+\left(u_{2,3}-u_{3,2}\right)^{2}+\left(u_{3,1}-u_{1,3}\right)^{2}+\lambda_{3}{ }^{2} \lambda_{1}\left(u_{1,2}-u_{2,1}\right)^{2} \\
\left.+\lambda_{1} \lambda_{3}\left(\lambda_{1}-\lambda_{3}\right)\left[\left(u_{1,2}\right)^{2}+\left(u_{2,1}\right)^{2}\right]\right\} d V .
\end{gathered}
$$

We note that for the state (b(ii)) in Sec. $3, \lambda_{3}<\frac{1}{3} T / C$ and accordingly, from $(3.10)_{2}$, $\lambda_{1}>\frac{2}{3} T / C$, so that $\lambda_{1}>2 \lambda_{3}$. In this case the coefficients in the integrand of (4.8) of each of the squares involving $u_{i, A}(i \neq A)$ are positive. Thus, for this case, if the deformation is stable with respect to superposed infinitesimal pure homogeneous deformations having the same principal directions as the basic pure homogeneous deformation, it will be stable with respect to an arbitrary superposed infinitesimal homogeneous deformation.

We shall show, in accord with the result of Rivlin [1], that the state (b(ii)) is in fact stable with respect to superposed infinitesimal pure homogeneous deformations having the same principal directions as the basic pure homogeneous deformation. To do this we take $u_{i, A}=0(i \neq A)$ in (4.8) and investigate the sign of the resulting expression for $\delta^{2} \Phi$. We obtain, for $\delta^{2} \Phi$,

$$
\delta^{2} \Phi=\frac{1}{2} C \int_{V}\left\{\left(u_{1,1}+u_{2,2}+u_{3,3}\right)^{2}-2\left(1-\lambda_{1} \lambda_{3}^{2}\right) u_{1,1} u_{2,2}\right\} d V .
$$

For stability, $\delta^{2} \Phi>0$ for all $u_{1,1}, u_{2,2}, u_{3,3}$ satisfying the relation (2.8), which may now be rewritten as

$$
\frac{1}{\lambda_{1}}\left(u_{1,1}+u_{2,2}\right)+\frac{1}{\lambda_{3}} u_{3,3}=0 .
$$

Using (4.10) to eliminate $u_{3,3}$ from (4.9), we obtain, with (4.10) ${ }_{1}$,

$$
\delta^{2} \Phi=\frac{1}{2} C \int_{V} \lambda_{3}\left(\lambda_{1}-\lambda_{3}\right)\left\{\left(\lambda_{1}-\lambda_{3}\right)\left[\left(u_{1,1}\right)^{2}+\left(u_{2,2}\right)^{2}\right]-2 \lambda_{3} u_{1,1} u_{2,2}\right\} d V .
$$

The condition $\delta^{2} \Phi>0$ is satisfied for arbitrary choice of $u_{1,1}$ and $u_{2,2}$, if and only if $\lambda_{1}>2 \lambda_{3}$. It is therefore satisfied for the state ( $\mathrm{b}(\mathrm{ii})$ ), which is accordingly stable with respect to all superposed infinitesimal homogeneous deformations. The condition $\lambda_{1}>2 \lambda_{3}$ is violated for the state of deformation $(b(v))$, which is therefore unstable.

Precisely analogous considerations apply to the states (b(iii)) and (b(iv)), which are accordingly stable with respect to all superposed infinitesimal homogeneous deformations, and to states $(\mathrm{b}(\mathrm{vi}))$ and $(\mathrm{b}(\mathrm{vii}))$, which are unstable.

\section{References}

[1] R. S. Rivlin, Phil. Trans. Roy. Soc. A 240, 491 (1948)

[2] R. Hill, J. Mech. and Phys. of Solids 5, 229 (1957)

[3] A. E. Green and J. E. Adkins, Large elastic deformations, Clarendon Press, Oxford (1960)

[4] M. F. Beatty, Inst. J. Solids Structures 3, 23 (1967) 